\title{
A defect in the NOG gene increases susceptibility to spontaneous superficial chronic corneal epithelial defects (SCCED) in boxer dogs
}

Kathryn M. Meurs ${ }^{1,2}$, Keith Montgomery ${ }^{1,2}$, Steven G. Friedenberg ${ }^{3}$, Brian Williams $s^{1,2}$ and Brian C. Gilger ${ }^{1,2^{*}}$

\begin{abstract}
Background: Superficial chronic corneal epithelial defects (SCCEDs) are spontaneous corneal defects in dogs that share many clinical and pathologic characteristics to recurrent corneal erosions (RCE) in humans. Boxer dogs are predisposed to SCCEDs, therefore a search for a genetic defect was performed to explain this susceptibility. DNA was extracted from blood collected from Boxer dogs with and without SCCEDs followed by whole genome sequencing (WGS). RNA sequencing of corneal tissue and immunostaining of corneal sections from affected SCCED Boxer dogs with a deletion in the NOG gene and affected non-Boxer dogs without the deletion were performed.

Results: A 30 base pair deletion at a splice site in Noggin (NOG) (Chr 9:31453999) was identified by WGS and was significantly associated $(P<0.0001)$ with Boxer SCCEDs compared to unaffected non-Boxer dogs. NOG, BMP4, MMP13, and NCAM1 all had significant fold reductions in expression and SHH was significantly increased in Boxers with the NOG deletion as identified by RNA-Seq. Corneal IHC from NOG deletion dogs with SCCEDs had lower NOG and significantly higher scores of BMP2.
\end{abstract}

Conclusions: Many Boxer dogs with SCCED have a genetic defect in NOG. NOG is a constitutive protein in the cornea which is a potent inhibitor of BMP, which likely regulate limbal epithelial progenitor cells (LEPC).

Dysregulation of LEPC may play a role in the pathogenesis of RCE.

Keywords: Corneal ulcer, Chronic, Superficial, Recurrent erosion, NOG, Boxer

\section{Background}

Superficial chronic corneal epithelial defects (SCCEDs), also known as indolent corneal ulcers, are spontaneous corneal defects in dogs that share many clinical, pathophysiologic, and pathologic characteristics with recurrent corneal erosions (RCE) in humans [1-3]. These similarities include a spontaneous and recurrent nature, associated ocular discomfort, epithelial loss with a non-adherent

\footnotetext{
* Correspondence: bgilger@ncsu.edu

${ }^{1}$ Clinical Sciences, North Carolina State University, 1060 William Moore Drive, Raleigh, North Carolina 27613, USA

${ }^{2}$ Present address: Upstate Veterinary Specialties, Latham, NY, USA

Full list of author information is available at the end of the article
}

epithelial border, a paracentral lesion location, and a lack of infectious organisms [1-6]. Morphologic similarities also include a reduced adhesion of the corneal epithelium to the underlying extracellular matrix, a deficient epithelial basement membrane, absence and abnormality of basal epithelial cell hemidesmosomes, and a loss of anchoring fibrils from the basal epithelium to the stroma $[2,3,6]$. Furthermore, a reduction of corneal innervation was identified in dogs with SCCEDs, thus resembling neurotrophic corneal ulcers in humans [1]. RCEs and SCCEDs may be primarily the result of a defect in the epithelial basement membrane or poor epithelial healing from complete or

(C) The Author(s). 2021 Open Access This article is licensed under a Creative Commons Attribution 4.0 International License, which permits use, sharing, adaptation, distribution and reproduction in any medium or format, as long as you give appropriate credit to the original author(s) and the source, provide a link to the Creative Commons licence, and indicate if changes were made. The images or other third party material in this article are included in the article's Creative Commons licence, unless indicated otherwise in a credit line to the material. If material is not included in the article's Creative Commons licence and your intended use is not permitted by statutory regulation or exceeds the permitted use, you will need to obtain permission directly from the copyright holder. To view a copy of this licence, visit http://creativecommons.org/licenses/by/4.0/. The Creative Commons Public Domain Dedication waiver (http://creativecommons.org/publicdomain/zero/1.0/) applies to the data made available in this article, unless otherwise stated in a credit line to the data. 
partial limbal stem cell or limbal progenitor cell deficiencies [7-9]. RCEs may also be secondary to corneal degenerative diseases, dry eye, eyelid abnormalities, previous corneal surgery, and systemic diseases, such as diabetes, among other causes $[3,6]$.

Treatment of SCCEDs or RCEs remains challenging and therapies for these chronic, painful conditions have not changed substantially in the past several decades. Conservative treatments, such as epithelial debridement, topical antibiotics, and artificial tears are commonly used but rarely effective. Unresponsive patients may benefit from use of therapeutic bandage contact lenses, amnion membrane grafts, matrix metalloproteinase (MMP) inhibitors (topical or systemic), and/or topical serum eye drops. Diamond burr debridement, anterior stromal puncture, alcohol delamination, or superficial keratectomy are used in resistant cases, especially when the patient has suspected epithelial basement membrane dystrophies [3-6, 10-15]. Effective treatments that target the underlying cause of both SCCEDs and RCEs remain elusive.

Boxer dogs are highly predisposed to development of SCCEDs, however, the underlying cause is unknown [4, 16-18]. To determine if there is an underlying genetic abnormality to describe this predisposition, and to investigate the underlying pathogenesis of SCCEDs and RCEs (i.e., using the Boxer dog as a naturally-occurring model for RCEs), a search for a genetic defect was performed in Boxer dogs. Using whole genome sequencing (WGS), we identified a 30-base-pair deletion at a splice site in the noggin gene (NOG), which was significantly associated $(P<0.0001)$ with Boxer dogs with SCCEDs compared to unaffected non-Boxer dogs. Furthermore, RNA sequencing of corneal samples and immunostaining of keratectomy specimens from SCCED Boxer dogs deficient in NOG revealed reduced expression of NOG and alteration of RNA expression of several factors in the BMP signaling pathway compared to non-Boxer SCCED dogs that were wild type (WT) for the NOG deletion. These results suggest that NOG deficiency and effect on the BMP signaling pathway may impact limbal cell progenitor cells and play a role in SCCEDs and RCEs.

\section{Methods}

Use of animals in this study adhered to the Association for Research in Vision and Ophthalmology statement on the use of animals in ophthalmic and vision research. The animal use protocol was approved and monitored by the North Carolina State University Institutional Animal Care and Use Committee (IACUC) (Protocol \# 18164-0). The North Carolina State University Veterinary Hospital Board approved the protocol for evaluation of clinical patients in this study. Owners of dogs signed and provided informed consent for all sample collections and data collection. The study was carried out in compliance with the ARRIVE guidelines.

\section{Animal selection/phenotyping}

Whole blood samples were collected from Boxer dogs either with, or having a documented history (clinical cases of the NC State Veterinary Teaching Hospital Ophthalmology service) of having SCCED, and Boxer dogs over 10 years of age (clinical cases of the NC State Veterinary Teaching Hospital Cardiology service). with no known history of a SCCED and no evidence of previous corneal disease as examined by an NC State University veterinary ophthalmologist. All blood was collected in EDTA tubes and DNA was extracted using the standard protocol of the DNeasy Blood and Tissue Kit (Qiagen, Germantown, MD).

\section{Whole genome sequencing}

Samples from 8 SCCED Boxer dogs were submitted for library preparation and whole genome sequencing, using a 150 base pair (bp) paired-end read configuration on an Illumina HiSeq 4000 high-throughput sequencing system (Genewiz LLC, South Plainfield, NJ).

Variant calling from next-generation sequencing data was performed using a standardized bioinformatics pipeline for all samples, as described previously [19]. Sequence reads were trimmed using Trimmomatic 0.32 to a minimum phred-scaled base quality score of 30 at the start and end of each read, with a minimum read length of $70 \mathrm{bp}$, and aligned to the canFam 3 reference sequence using BWA 0.7.13 [20-22]. Aligned reads were prepared for analysis using Picard Tools 2.8 (http://broadinstitute. github.io/picard) and GATK 3.7 following best practices for base quality score recalibration and indel realignment (Broad Institute, Cambridge, MA, USA) [23, 24]. Variant calls were made using GATK's HaplotypeCaller walker, and variant quality score recalibration (VQSR) was performed using sites from dbSNP 146 and the Illumina $174 \mathrm{~K}$ CanineHD BeadChip as training resources. A VQSR tranche sensitivity cutoff of $99.9 \%$ to SNPs and 99\% to indels was used for downstream analyses; genotype calls with a phred-scaled quality score $<20$ were flagged but not removed from the variant callset.

Variants (heterozygous or homozygous) present in at least $7 / 8$ affected Boxers were identified. The resulting variants were then filtered against a previously established database of variants from 84 non-Boxer dogs of 17 different dog breeds without a known risk for development of SCCED (https://cidd.discoveryspace.ca/). (Supplemental data Table 1) Any variants with a minor allele frequency greater than $1 \%$ in the non-Boxer dogs were removed. The remaining variants were categorized by Variant Effect Predictor 91 (https://useast.ensembl. org/info/docs/tools/vep/index.html) and prioritized by 
their functional impact (e.g., stop codon, frameshift, change in amino acid, etc.). They were manually curated for potential role in corneal disease and corneal wound healing, such as bone morphogenetic proteins (BMPs), transforming growth factor beta superfamily, noggin, etc. Missense variants were evaluated for genomic functional significance with Polyphen (http://genetics.bwh.harvard. edu/pph2/), SIFT (http://sift.jcvi.org/) and Provean (http://provean.jcvi.org/index.php). In Silico Splice Site Analysis was performed with Human Slice Site Finder (http://www.umd.be/HSF/) to predict the potential impact of splice site changes.

Variants involved with genes associated with a potential role in corneal disease and/or corneal wound healing were prioritized based on the variant's impact on the gene (deleterious missense, stop/start gained or lost, inframe deletion, frameshift) and were pursued with Sanger Sequencing in the additional affected (62) Boxer dogs and apparently unaffected (25) Boxer dogs and tested for allelic association with SCCED using a Fisher's exact test. A $p$-value of $<0.05$ was considered significant.

Variants of strongest interest were subsequently filtered against a larger established canine database of 391 non-Boxer dogs of 53 different breeds to assess variant prevalence in the canine population.

\section{RNA library preparation and sequencing}

To assess RNA expression of genes in corneal tissue associated with SCCED, total RNA was extracted from corneal tissue gently debrided from four SCCED Boxer dogs with the NOG deletion and three affected nonBoxer dogs without the NOG deletion (Boston Terrier (2), Pomeranian) as has been described previously [25]. Using the Qiagen RNeasy Plus Universal mini kit (Qiagen, Germantown, MD) RNA was quantified using Qubit 2.0 Fluorometer (Life Technologies, Carlsbad, CA) and RNA integrity was checked with Agilent TapeStation (Agilent Technologies, Palo Alto, CA). RNA library preparation, sequencing, and initial bioinformatics analysis was conducted at GENEWIZ, LLC (South Plainfield, NJ). The RNA sequencing library preparation was performed with the NEBNext Ultra RNA Library Prep Kit (NEB, Ipswich, MA, USA) for Illumina (San Diego, CA) followed by manufacturer's instructions. The sequencing library was validated on an Agilent TapeStation (Agilent Technologies, Palo Alto, CA, USA), and quantified with a Qubit 2.0 Fluorometer (Invitrogen, Carlsbad, CA) as well as by quantitative PCR (KAPA Biosystems, Wilmington, MA, USA). The sequencing libraries were clustered on one lane of a flowcell. After clustering, the flowcell was loaded on the Illumina HiSeq 4000 high-throughput sequencing system instrument according to manufacturer's instructions. The samples were sequenced using a $2 \times 150$ Paired End (PE) configuration. Image analysis and base calling were conducted by the HiSeq Control Software. Raw sequence data (.bcl files) generated from Illumina HiSeq was converted into fastq files and de-multiplexed using Illumina's bcl2fastq 2.17 software. One mis-match was allowed for index sequence identification.

\section{RNA-Seq analysis}

The datasets and transcript counts were estimated using Salmon (version 0.11.2) in quasi-mapping mode against dog reference genome CanFam3.1 with default settings [26]. Salmon estimated counters were summarized to gene level using the tximport package in $\mathrm{R}$ ( $\mathrm{v}$ 3.4.4) for use with DESeq2 [27]. DESeq2 was used for differential expression testing. Differential gene expression analysis was performed by comparing samples from Boxer SCCE D cases to non-Boxer SCCED cases. A cutoff of $\mid \log _{2}$ fold change $\mid>1.5$ was considered significant [28].

Ingenuity Pathway Analysis (IPA) (Qiagen, Redwood CA) was used to evaluate key biologic pathways involving NOG to identify genes that could be impacted by altered NOG for further assessment with RNA Seq and immunohistochemical analysis.

\section{Immunohistochemical analysis of corneal specimens from SCCED dogs}

Keratectomies were performed to treat the SCCED in five Boxer dogs with the NOG deletion and three affected non-Boxer dogs without the NOG deletion. Corneal keratectomy specimens were preserved for future analysis and were fixed in $4 \%$ buffered paraformaldehyde overnight at $4{ }^{\circ} \mathrm{C}$ and then transferred to $70 \%$ ethanol before being embedded in paraffin. Tissues were sectioned at $5 \mu \mathrm{m}$ and stained with hematoxylin and eosin. Immunofluorescence was performed following a previously described method with immunohistochemistry antibodies selected for the gene of interest and genes in the biologic pathway predicted to be impacted by altered gene function [29]. In short, sections were deparaffinized by incubating the slides two times in xylene for $10 \mathrm{~min}$ (min) each, followed by immersing the slides sequentially in two rounds of 100\% (3 min each), $95 \%$ ( $1 \mathrm{~min}$ ), and $80 \%(1 \mathrm{~min})$ ethanol solutions, and finally in water for $5 \mathrm{~min}$. Antigen retrieval procedure was performed by heating the slides to $95^{\circ} \mathrm{C}$ in citrate-based ( $\mathrm{pH}$ 6.0) antigen unmasking buffer (Vector Laboratories) before staining. Non-specific staining was blocked by using PBS containing $10 \%$ of normal goat serum, $0.025 \%$ Triton X100 plus $1 \%$ BSA before overnight incubation with the primary antibody. The primary antibodies included rabbit polyclonal NOG 1:100 (Abcam ab16054); rabbit polyclonal BMP2 (Novus NBP1-19751SS), rabbit polyclonal BMP4 (Abnova PAB3673). Negative controls were performed as described above but without use of a 
primary antibody. After the staining, slides were mounted and counter stained with ProLong ${ }^{\mathrm{Tm}}$ Diamond Antifade Mountant with DAPI (p36971, Invitrogen) and observed by Olympus IX83 Fluorescence Microscope (Olympus, Tokyo, Japan) or Zeiss LSM 780 inverted confocal microscope. Intensity and distribution of IHC staining was scored by two, blinded, experienced examiners using the following scoring scheme; $0=$ no staining; 1 = slight, focal corneal staining; 2 = slight, diffuse staining; 3 =moderate intensity, focal to diffuse; $4=$ high intensity, diffuse staining. Results of the two observers were averaged to provide a final score for each specimen. Pairwise Wilcoxon (Mann-Whitney tests) were performed to evaluate for group differences in IHC scores using JMP version 14.0 (SAS Institute Cary, NC).

\section{Results}

Animal selection/phenotyping

Seventy Boxer dogs either with, or having a documented history of SCCED and twenty-five Boxer dogs at least 10 years of age with no known history of SCCED were identified.

\section{Whole genome sequencing}

After filtering, 5013 variants were identified in at least 7 of 8 affected Boxers and in less than $1 \%$ of the 84 nonBoxer dogs from 17 different dog breeds. After filtering for genes identified as those likely to have a possible role in corneal disease and/or corneal wound healing, eight variants remained in five genes, KIF5C, NOTCH1, EMCS, Serpine2 and NOG. (Table 1) These variants were all pursued by Sanger sequencing. The variants in KIF5C were all predicted to be low impact (intronic, non-splice site SNPs) but were pursued anyway because of the apparent involvement of the gene in the cornea. All remaining variants were in genes without a clear likely role in corneal disease and/or corneal wound healing and were SNPs or small indels located in a $3^{\prime}$ or $5^{\prime}$ untranslated, upstream, downstream or intronic region. These were not evaluated further.
These eight variants were further evaluated by Sanger Sequencing. The variants in KIF5C, NOTCH1 and EMCS were inconsistently found in the affected dog population and did not have a statistically significant association with SCCED. In contrast, the variants in Serpine 2 and NOG were found in all of the SCCED Boxer dogs but also were found in 23 of 25 , and 24 of 25 of the Boxers without SCCED, respectively.

When evaluated against the larger canine database of 391 non-Boxer dogs of 53 different breeds, the SERP INE2 variant was also found in 22 dogs of 11 breeds (5\% of the overall dog population) while the NOG variant was found in only 4 dogs of one additional breed (Yorkshire Terrier) (less than 1\% of the overall dog population).

The NOG variant was a 30 base pair deletion at a splice site (Chromosome 9:31,453,999-31,454,029, ENSC AFT00000047287.1:c.46 + 13_46 +

42delGTGTGTGTGTGTGTGTGAGTGTGTGTGTGT). In silico splice site analysis predicted the NOG variant to disrupt a splice site as well as an enhancer motif site.

\section{RNA-Seq}

With a cutoff of $\mid \log _{2}$ fold change $\mid>1.5$ considered significant, Serpine2, was not differentially expressed $(0.015$ $\log _{2}$ fold) between the SCCED groups while.

NOG expression was reduced (2.8 $\log _{2}$ fold). (Supplemental Data Table 2) Four genes within the BMP Signaling Pathway which were expressed in the cornea were found to be differentially expressed including BMP4, MMP13, NCAM1 (all reduced) and SHH (increased) (Table 2) (Fig. 1A-E).

\section{Corneal immunohistochemistry}

Samples from Boxer SCCED dogs with the NOG deletion had a significantly reduced IHC score $(p=0.03)$ compared to mean IHC scores from corneal samples from non-Boxer SCCED dogs without the NOG deletion (Fig. 2). Keratectomy specimens from Boxer SCCED dogs had significantly higher BMP2 $(p=0.01)$ scores

Table 1 Variants identified in Boxers dogs with SCCED in genes likely to have a possible role in corneal disease and corneal wound healing

\begin{tabular}{lllll}
\hline Chromosomal location & Gene & Reference allele & Variant allele & Gene location \\
\hline 19: 50605253 & KIF5C & C & A & Intronic \\
19: 50602128 & KIF5C & A & T & Intronic \\
19: 50543167 & KIF5C & T & A & Intronic \\
19: 50546726 & KIF5C & C & Intronic \\
32: 22284093 & EMCS & A & G & Missense \\
37: 29701267 & Serpine 2 & A & A & Splice Site \\
$9: 49015931$ & NOTCH1 & G & & Missense \\
$9: 31453999$ & NOG & gtgtgtgtgtgtgt & Deletion \\
\hline
\end{tabular}


Table 2 Differential expression of NOG and genes that NOG regulates in the BMP Signaling Pathway from SCCED Boxer dogs with NOG deletion compared to SCCED non-Boxer without the NOG deletion

\begin{tabular}{ll}
\hline Gene & Fold Change $^{\mathbf{a}}$ \\
\hline NOG & -2.8 \\
Alp & Not expressed \\
BMP & Not expressed \\
BMP2 & NS \\
BMP4 & -1.6 \\
FGF8 & Not expressed \\
LEF1 & NS \\
MMP13 & -2.3 \\
NCAM1 & -4.4 \\
RUNX2 & Not expressed \\
SHH & 3.9 \\
SMAD1 & Not expressed \\
VEGFA & NS \\
\hline
\end{tabular}

${ }^{a}$ Not expressed indicates that measurable amounts were not detected in the sample, NS indicates that the fold change was less than 1.5

than corneal samples from non-Boxer SCCED dogs (Wilcoxon Test) although it was not found to be significantly different by RNA Seq. (Supplemental data Table 2) BMP4 appeared to be reduced but the differences were not significant by IHC (Fig. 2 A-H).

\section{Discussion}

Our data have demonstrated that many Boxer dogs with superficial nonhealing corneal ulcers have a genetic defect in the NOG gene (a 30 base pair deletion at a splice site in NOG [Chromosome 9:31,453,999-31,454,029]), which encodes the protein noggin, a constitutivelysecreted, $46 \mathrm{kDa}$, disulfide-linked homodimer consisting of two, 206 amino acid polypeptide chains [30]. Not only was the genetic NOG defect highly associated with SCCED Boxer dogs, but these NOG deficient dogs also had greater than two fold reduction of corneal epithelial noggin RNA signal and significantly reduced noggin staining in the stroma of keratectomy samples compared to $N^{+} G^{+}$SCCED dogs. Noggin is involved in the bone morphogenetic protein (BMP) pathway where it serves as a potent inhibitor of BMP of the transforming growth factor- $\beta$ superfamily. Noggin binds to BMPs receptor complex (BMPR-I) preventing its activation [30, 31]. In the Boxer dogs with SCCED evaluated here the reduction of Noggin was identified, as well as alterations in expression of several other key factors in the BMP Signaling pathway including NCAM1, MMP13 and SHH (Table 2). Noggin regulation should increase expression of NCAM1 and MMP13, and decrease expression of $\mathrm{SHH}$. In the dogs reported here, we identified decreased
NCAM1 and MMP13 and increased SHH as would be expected with reduced levels of NOG (Table 3). Specifically, noggin inhibits BMP2, 4, 5, 7, 13 and 14, but leaves BMP3, 6, 9, 10 and 15 signaling unaffected [30]. Both BMP2 and BMP4 are also expected to be inhibited by NOG. Here we found BMP2 to have a measurable increase according to the corneal stroma IHC analysis, although not in the epithelium by RNA, as would be expected with a reduction in NOG. BMP4, was decreased at the RNA level. BMP4 is both negatively regulated by NOG, as well as having a role in regulating NOG and its response to reduced NOG may be more complex.

This study demonstrated a significant association between a genetic NOG defect, decreased tissue noggin, alterations in the BMP signaling pathway and delayed corneal epithelial wound healing in dogs diagnosed with SCCED. Several studies have also observed reduced keratinocyte proliferation and delayed dermal wound healing associated with reduced noggin and increased BMP activity [30, 32]. BMPs, BMP receptors, and noggin have been shown to be expressed in human corneal epithelial cells and keratocytes, and they likely regulate limbal stem cells and corneal epithelial wound healing [33, 34]. Limbal stem cells reside in a cellular complex called the stem cell niche [35], consisting of the limbal epithelial progenitor cells (LEPC) and limbal niche cells (LNC), the latter of which controls self-renewal and fate decisions of LEPCs partially through production of noggin [34]. Noggin suppresses BMPs in LEPCs through suppression of the SMAD signaling pathway and activates canonical Wnt signaling which is correlated with clonal growth of LEPCs [34]. Therefore, Noggin deficiency would allow BMP to bind to BMPR-I, initiate SMAD signaling, reduce Wnt signaling, and suppress LEPC growth, all of which may contribute to development of SCCEDs.

Although BMPs and Noggin effect on corneal wound healing has not been well described, BMPs and noggin have been shown to have an effect on dermal wound repair and healing. BMP2 and BMP4 have been demonstrated to reduce keratinocyte proliferation [36, 37] and transgenic mice overexpressing SMAD1 (part of the BMP signaling cascade) have delayed dermal wound healing [32]. Furthermore, exogenous topical administration of BMP4 and 7 to a human ex vivo dermal wound healing model impaired epithelial wound closure [32]. Overall, the effect of BMPs is to suppress keratinocyte proliferation and increase apoptosis in the dermal wound epithelium, thus negatively regulate keratinocyte proliferation and migration during wound healing [32]. Alternatively, noggin has been shown to improve dermal wound healing. Transgenic mice that overexpress noggin have accelerated dermal wound healing, increased 


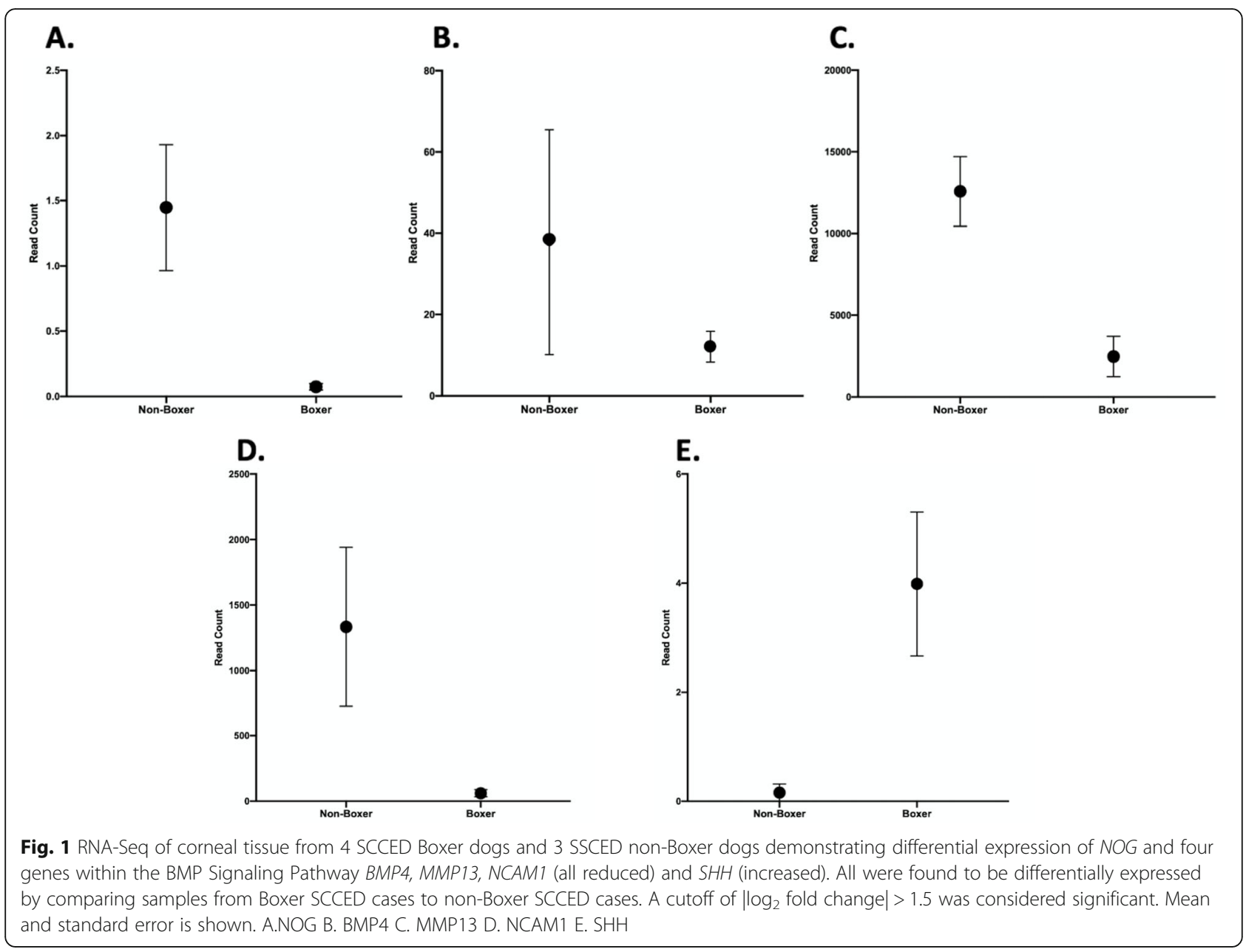

keratinocyte proliferation, and increased wound vasculature compared to wild type mice [32]. Exogenous topical treatment with noggin to a human ex vivo dermal wound healing model resulted in accelerated epithelial wound closure [32]. Therefore, inhibiting BMPs or providing exogenous noggin may improve dermal and corneal epithelialization; and provide a potential therapeutic for SCCED and chronic recurrent erosions.

NOG mutations have been reported in humans as an autosomal dominant disease with variable phenotype, commonly referred to as symphalangism spectrum disorder (NOG-SSD), involving 5 main autosomal dominant syndromes: (1) proximal symphalangism; (2) multiple synostoses syndrome 1 ; (3) stapes ankylosis with broad thumbs and toes; (4) tarsal-carpal coalition syndrome; and (5) brachydactyly type B2 [38-40]. Other than hyperopia and strabismus, which is common, ocular abnormalities have not been reported in these patients [40]. Boxer dogs with SCCEDs have not been reported to have orthopedic or joint disorders, nor did the dogs with the NOG deletion included in this study.
In the dogs evaluated here, Boxer dogs without a known SCCED history also commonly had the NOG deletion. If noggin deficiency is associated with suppression of LEPC growth one might hypothesize that many Boxers will have a normal phenotype until they suffer a corneal abrasion and at that point the NOG deletion and its impact on the BMP signaling pathway could prevent the LEPC growth and normal healing that would occur in a dog without this deletion. Only at that time would the SCCED phenotype become apparent. Additionally, since the deletion is not one that would shorten life span or impact successful reproduction it would not have been naturally selected against and could be quite widespread within the breed. However, the pathogenesis of SCCED in dogs has been reported to a reduced adhesion of the corneal epithelium to the underlying corneal extracellular matrix and not a result of reduced corneal epithelial growth $[1,2,17]$. It is possible that Noggin deficiency may negatively regulate stromal keratinocytes, as seen in dermal wound healing [32], leading to changes in extracellular matricies or epithelial basement membrane in the cornea. These changes may then lead to 


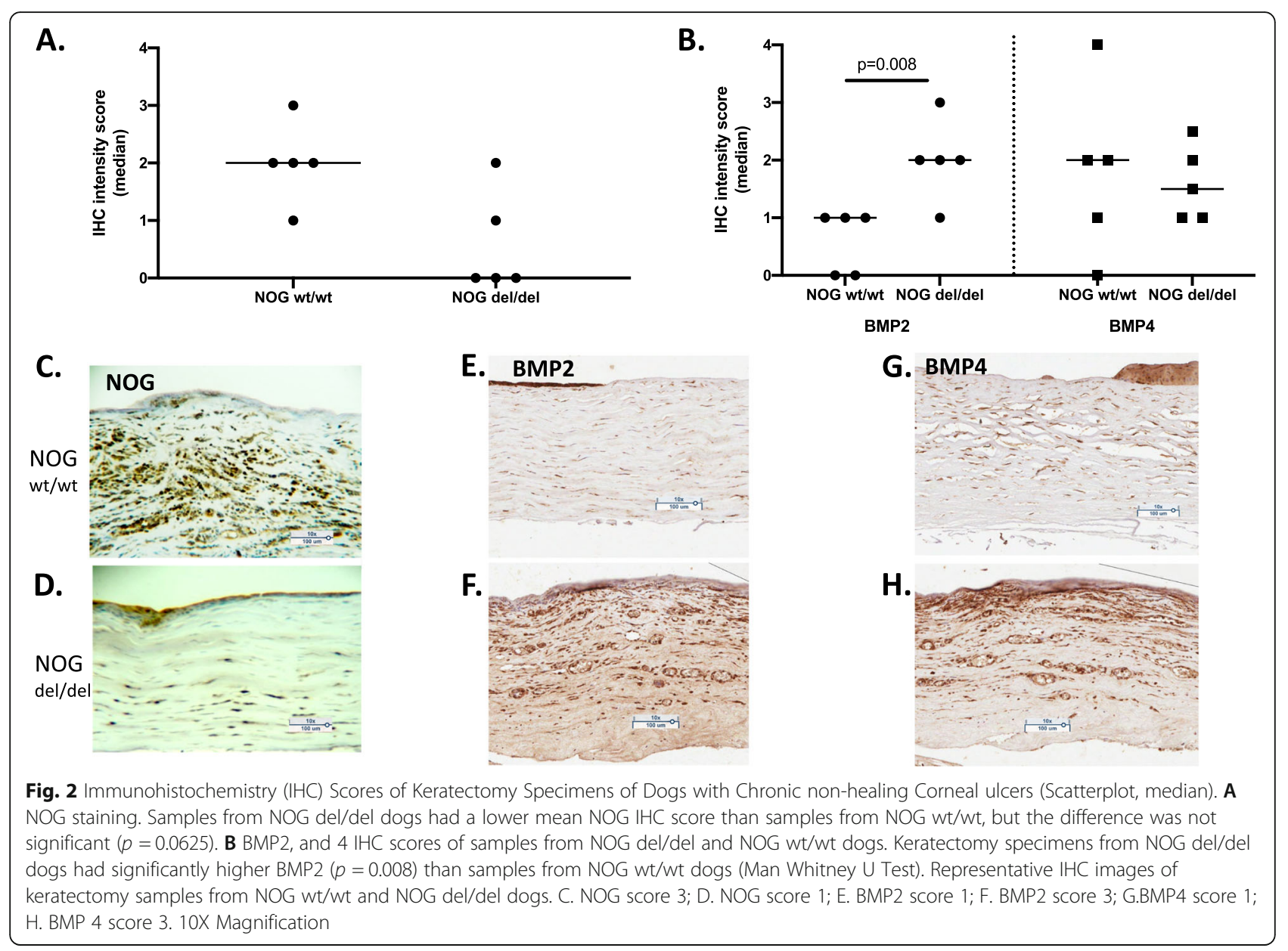

reduced adhesion of the corneal epithelium to the underlying extracellular matrix and corneal stroma in SCCED [1, 2, 17]. Further study of the role of Noggin and the effects of its deficiency o on corneal wound healing and on the development of SCCED in dogs is needed.

In this study, using whole genome sequencing (WGS), we identified a 30 base pair deletion at a splice site in the noggin gene (NOG) which was significantly associated with Boxer dogs with SCCEDs compared to nonBoxer dogs. Furthermore, RNA sequencing of corneal samples and immunostaining of keratectomy specimens

Table 3 Immunohistochemistry scoring of NOG and genes that NOG regulates in the BMP Signaling Pathway from SCCED Boxer dogs with NOG deletion compared to SCCED non-Boxer without the NOG deletion

\begin{tabular}{llll}
\hline & $\begin{array}{l}\text { NOG }^{-} \text {IHC score } \\
(\text { Mean } \pm \text { SD) }\end{array}$ & $\begin{array}{l}\text { NOG WT score } \\
(\text { Mean } \pm \text { SD) }\end{array}$ & $P$ value \\
\hline NOG & $0.6 \pm 0.9$ & $2.0 \pm 0.7$ & 0.03 \\
BMP4 & $1.6 \pm 0.7$ & $2.0 \pm 1.6$ & 0.62 \\
BMP2 & $2.0 \pm 0.7$ & $0.6 \pm 0.5$ & 0.01 \\
\hline
\end{tabular}

from SCCED Boxer dogs deficient in NOG revealed reduced RNA signal and expression of NOG, respectively, and altered expression of BMP signaling pathway factors compared to WT dogs. A limitation of this study is that we compared corneal tissue from Boxer dogs with SCCE Ds to non-Boxer dog with SCCEDs, rather than comparing it to corneal tissue from unaffected control dogs. Since SCCEDs are likely to have RNA and protein expression alterations that occur as a consequence of the corneal ulcer, comparison to unaffected corneal tissue could have emphasized changes that occur due to SCCE $\mathrm{D}$ rather than the underlying cause. Finally, additional analysis of the NOG RNA for alignment and transcript analysis would have been useful but unfortunately the quality and quantity of NOG RNA from debrided cells was quite limited and prevented additional transcript analysis.

\section{Conclusions}

Many Boxer dogs with SCCED have a genetic defect in NOG. NOG is a constitutive protein in the cornea which is a potent inhibitor of BMP, which likely regulate limbal epithelial progenitor cells (LEPC). Dysregulation of 
LEPC may play a role in the pathogenesis of RCE. These results suggest that NOG deficiency play a role in SCCE Ds and RCEs. Further study of this defect in the pathogenesis of SCCED and RCE appears warranted.

\begin{abstract}
Abbreviations
BMP: Bone morphogenic protein; Chr: Chromosome; IACUC: Institutional animal care and use committee; ICH: Immunohistochemistry; LEPC: Limbal epithelial progenitor cells; NOG: Noggin; RCE: Recurrent corneal erosions; SCCED: Superficial chronic corneal epithelial defects; WGS: Whole genome sequencing
\end{abstract}

\section{Supplementary Information}

The online version contains supplementary material available at https://doi. org/10.1186/s12917-021-02955-1.

\section{Additional file 1}

\section{Acknowledgements}

The authors thank Damian Launer, Melissa Hamman, Erin Barr, and Beth Salmon for technical support. The authors also thank the University of North Carolina Histology Core for processing of the corneal histology and immunohistochemistry.

\section{Authors' contributions}

KMM designed the study, analyzed and interpreted the data, and wrote manuscript; KM designed the study and revised the manuscript; SG analyzed data and revised the manuscript; BW analyzed data and revised the manuscript; and BG collected samples, analyzed and interpreted the data, and wrote manuscript. All authors read and approved the final manuscript.

\section{Funding}

Grants: American Kennel Club - Canine Health Fund.

\section{Availability of data and materials}

The datasets used and/or analyzed during the current study are available from the corresponding author on reasonable request.

\section{Declarations}

\section{Ethics approval and consent to participate}

Use of animals in this study adhered to the Association for Research in Vision and Ophthalmology (ARVO) statement on the use of animals in ophthalmic and vision research. The animal use protocol was approved and monitored by the North Carolina State University Institutional Animal Care and Use Committee (IACUC) (Protocol \# 18-164-0). The North Carolina State University Veterinary Hospital Board approved the protocol for evaluation of clinical patients in this study. Owners of dogs signed and provided informed consent for all sample collections and data collection. The study was carried out in compliance with the ARRIVE guidelines.

\section{Consent for publication}

All authors consent to publication.

\section{Competing interests}

The authors declare that they have no competing interests.

\section{Author details}

${ }^{1}$ Clinical Sciences, North Carolina State University, 1060 William Moore Drive, Raleigh, North Carolina 27613, USA. ${ }^{2}$ Present address: Upstate Veterinary Specialties, Latham, NY, USA. ${ }^{3}$ Veterinary Clinical Sciences, University of Minnesota, St. Paul, MN, USA.
Received: 31 January 2021 Accepted: 1 July 2021

Published online: 26 July 2021

\section{References}

1. Murphy CJ, Marfurt CF, McDermott A, Bentley E, Abrams GA, Reid TW, et al. Spontaneous chronic corneal epithelial defects (SCCED) in dogs: clinical features, innervation, and effect of topical SP, with or without IGF-1. Investig Ophthalmol Vis Sci. 2001:42:2252-61.

2. Bentley E, Abrams GA, Covitz D, Cook CS, Fischer CA, Hacker D, et al. Morphology and immunohistochemistry of spontaneous chronic corneal epithelial defects (SCCED) in dogs. Investig Ophthalmol Vis Sci. 2001:42: 2262-9.

3. Das S, Seitz B. Recurrent corneal Erosion syndrome. Surv Ophthalmol. 2008; 53:3-15.

4. Chandler HL, Gemensky-Metzler AJ, Bras ID, Robbin-Webb TE, Saville WJ, Colitz $\mathrm{CMH}$. In vivo effects of adjunctive tetracycline treatment on refractory corneal ulcers in dogs. J Am Vet Med Assoc. 2010;237:378-86.

5. Diez-Feijóo E, Grau AE, Abusleme El, Durán JA. Clinical presentation and causes of recurrent corneal erosion syndrome: Review of 100 patients. Cornea. 2014:33(6):571-5.

6. Miller DD, Hasan SA, Simmons NL, Stewart MW. Recurrent corneal erosion: a comprehensive review. Clin Ophthalmol. 2019:13:325-35.

7. Dua HS, Azuara-Blanco A. Limbal stem cells of the corneal epithelium. Surv Ophthalmol. 2000:44(5):415-25.

8. Le Q, Xu J, Deng SX. The diagnosis of limbal stem cell deficiency. Ocular surface. 2018. Ocul Surf. 2018:16(1):58-69.

9. Anderson DF, Ellies P, Pires RTF, Tseng SCG. Amniotic membrane transplantation for partial limbal stem cell deficiency. Br J Ophthalmol. 2001; 85(5):567-75.

10. Avni Zauberman N, Artornsombudh P, Elbaz U, Goldich Y, Rootman DS, Chan CC. Anterior stromal puncture for the treatment of recurrent cornea erosion syndrome: patient clinical features and outcomes. Am J Ophthalmol. 2014;157(2):273-9.

11. Laibson PR. Recurrent corneal erosions and epithelial basement membrane dystrophy. Eye Contact Lens. 2010;36(5):315-7.

12. Singh RP, Raj D, Pherwani A, Lagnado R, Abedin A, Eatamadi $H$, et al. Alcohol delamination of the corneal epithelium for recalcitrant recurrent corneal erosion syndrome: a prospective study of efficacy and safety. Br J Ophthalmol. 2007:91:908-11.

13. Tsai TY, Tsai TH, Hu FR, Hou YC. Recurrent corneal erosions treated with anterior stromal puncture by neodymium: yttrium-aluminum-garnet laser. Ophthalmology. 2009;116(7):1296-300.

14. Kaz Soong H, Farjo Q, Meyer RF, Sugar A. Diamond burr superficial keratectomy for recurrent corneal erosions. Br J Ophthalmol. 2002:86:296-8.

15. Gosling A, Labelle AL, Breaux CB. Management of spontaneous chronic corneal epithelial defects (SCCEDs) in dogs with diamond burr debridement and placement of a bandage contact lens. Vet Ophthalmol. 2013;16:83-8.

16. Roberts SR. Superficial indolent ulcer of the cornea in Boxer dogs. J Small Anim Prac; 6:111-115.

17. Chavkin MJ, Riis RC, Scherlie PH. Management of a persistent corneal erosion in a boxer dog. Cornell Vet. 1990;80(4):347-56.

18. Eaton JS, Hollingsworth SR, Holmberg BJ, Brown MH, Smith PJ, Maggs DJ. Effects of topically applied heterologous serum on reepithelialization rate of superficial chronic corneal epithelial defects in dogs. J am vet med Assoc. 2017. J Am Vet Med Assoc. 2017:250(9):1014-22.

19. Friedenberg SG, Meurs KM. Genotype imputation in the domestic dog. Mamm Genome. 2016:27(9-10):485-94.

20. Bolger AM, Lohse M, Usadel B. Trimmomatic: a flexible trimmer for Illumina sequence data. Bioinformatics. 2014:30:2114-20.

21. Li H, Durbin R. Fast and accurate short read alignment with burrowswheeler transform. Bioinformatics. 2009:25:1754-60.

22. Depristo MA, Banks E, Poplin RE, Garimella KV, Maguire JR, Hartl C, et al. A framework for variation discovery and genotyping using next- generation DNA sequencing data. Nat Genet. 2011:43:491-8.

23. McKenna A, Hanna M, Banks E, Sivachenko A, Cibulskis K. The genome analysis toolkit: a MapReduce framework for analyzing next-generation DNA sequencing data Aaron. Genome Res. 2010;20:1297-303.

24. Van Der Auwera G, Carneiro MO, Hartl C, Poplin R, Levy-moonshine A, Jordan T, et al. From FastQ data to high confidence varant calls: the Genonme analysis toolkit best practices pipeline. Curr Protoc Bioinformatics. 2013;43(1110):11.10.1-11.10.33. 
25. Tokuda Y, Okumura N, Komori Y, Hanada N, Tashiro K, Koizumi N, et al. Transcriptome dataset of human corneal endothelium based on ribosomal RNA-depleted RNA-Seq data. Sci Data. 2020;7:407.

26. Patro R, Duggal G, Love MI, Irizarry RA, Kingsford C. Salmon: fast and biasaware quantification of transcript expression using dual-phase inference. Nat Methods. 2017;14:417.

27. Soneson C, Love MI, Robinson MD. Differential analyses for RNA-seq transcript-level estimates improve gene-level inferences. F1000Research. 2015;4:1521.

28. Makhijani RK, Raut SA, Purohit HJ. Fold change based approach for identification of significant network markers in breast, lung and prostate cancer. IET Syst Biol. 2018;12:213-8.

29. Hirsch ML, Conatser LM, Smith SM, Salmon JH, Wu J, Buglak NE, et al. AAV vector.meditated expression of HLA-G reduces injury-induced corneal vascularization, immune cell infiltration, and fibrosis. Sci Rep. 2017;7(1): 17840.

30. Krause C, Guzman A, Knaus P. Noggin. Int J Biochem Cell Biol. 2011:43:47881. https://doi.org/10.1016/j.biocel.2011.01.007.

31. Wordinger RJ, Clark AF. Bone morphogenetic proteins and their receptors in the eye. Exp Biol Med. 2007;232:979-92.

32. Lewis CJ, Mardaryev AN, Poterlowicz K, Sharova TY, Aziz A, Sharpe DT, et al. Bone morphogenetic protein signaling suppresses wound-induced skin repair by inhibiting keratinocyte proliferation and migration. J Invest Dermatol. 2014;134:827-37. https://doi.org/10.1038/jid.2013.419.

33. Mohan RR, Kim WJ, Mohan RR, Chen L, Wilson SE. Bone morphogenic proteins 2 and 4 and their receptors in the adult human cornea. Invest Ophthalmol Vis Sci. 1998;39(13):2626-36.

34. Han B, Chen S-Y, Zhu Y-T, Tseng SCG. Integration of BMP/Wnt signaling to control clonal growth of limbal epithelial progenitor cells by niche cells. Stem Cell Res. 2014;12:562-73. https://doi.org/10.1016/j.scr.2014.01.003.

35. Stepp MA, Zieske JD. The corneal epithelial stem cell niche. Ocul Surf. 2005; 3:15-26. https://doi.org/10.1016/S1542-0124(12)70119-2.

36. Ahmed MI, Mardaryev AN, Lewis CJ, Sharov AA, Botchkareva NV. MicroRNA21 is an important downstream component of BMP signalling in epidermal keratinocytes. J Cell Sci. 2011;124(Pt 20):3399-404.

37. Sharov AA, Sharova TY, Mardaryev AN, Di Vignano AT, Atoyan R, Weiner L, et al. Bone morphogenetic protein signaling regulates the size of hair follicles and modulates the expression of cell cycle-associated genes. Proc Natl Acad Sci U S A. 2006:103(48):18166-71.

38. Takano K, Ogasawara N, Matsunaga T, Mutai H, Sakurai A, Ishikawa A, et al. A novel nonsense mutation in the NOG gene causes familial NOG-related symphalangism spectrum disorder. Hum Genome Var. 2016;3:16023.

39. Masuda S, Namba K, Mutai H, Usui S, Miyanaga Y, Kaneko H, et al. A mutation in the heparin-binding site of noggin as a novel mechanism of proximal symphalangism and conductive hearing loss. Biochem Biophys Res Commun. 2014:447(3):496-502.

40. Potti TA, Petty EM, Lesperance MM. A comprehensive review of reported heritable noggin-associated syndromes and proposed clinical utility of one broadly inclusive diagnostic term: NOG-related-symphalangism spectrum disorder (NOG-SSD). Hum Mutat. 2011;32:877-86. https://doi.org/10.1002/ humu.21515.

\section{Publisher's Note}

Springer Nature remains neutral with regard to jurisdictional claims in published maps and institutional affiliations.

Ready to submit your research? Choose BMC and benefit from:

- fast, convenient online submission

- thorough peer review by experienced researchers in your field

- rapid publication on acceptance

- support for research data, including large and complex data types

- gold Open Access which fosters wider collaboration and increased citations

- maximum visibility for your research: over $100 \mathrm{M}$ website views per year

At $\mathrm{BMC}$, research is always in progress.

Learn more biomedcentral.com/submissions 